\title{
The influence of saccadic eye movements on postural stability during standing on an unstable platform
}

\author{
Milena Vagaja* and Lucia Bizovska \\ Faculty of Physical Culture, Palacký University Olomouc, Olomouc, Czech Republic
}

Copyright: (C) 2019 M. Vagaja and L. Bizovska. This is an open access article licensed under the Creative Commons Attribution License (https://creativecommons.org/licenses/by/4.0/).

Background: Vision is seen as an important factor in maintaining balance, but different visual conditions, such as following a cue or looking in one direction may increase or decrease body sway. Contradicting results in earlier studies focusing on visual control of posture show the same eye movements' conditions have a different impact on postural stability. Objective: The aim of the study was to assess the influence of different eye movements on standing balance performance. Methods: Thirty-two healthy students (age $22.8 \pm 2.7$ years) participated in the study. Participants were asked to follow a stationary dot and moving dots in horizontal and vertical directions in low and high frequencies while standing on a stabilometer - an unstable platform which allows movement in the frontal plane. Results: There was no impact of different saccadic speeds $\left(p=.251\right.$, partial $\left.\eta^{2}=.044\right)$ nor different directions $(p=.287$, partial $\eta^{2}=.039$ ) on a postural sway in young adults. Conclusions: The postural stability is not influenced by visual stimuli in healthy young adults while maintaining balance on an unstable platform.

Keywords: postural stability, stabilometer, visual manipulation, eye tracker

\section{Introduction}

Motor control relies on the utilization of sensory information to regulate our movements (Latash, 2012; Schmidt \& Lee, 2011). It is important to determine position in an environment so the body can give the proper motor response, it is also necessary of the sensory systems to provide the correct orientation information into the central nervous system (Hammami, Behm, Chtara, Othman, \& Chaouachi, 2014; Han, Anson, Waddington, Adams, \& Liu, 2015; Redfern \& Furman, 1994). If the adjustment in the integration among the senses is interrupted, systems information differs one from another and the postural control is disturbed (Redfern, Yardley, \& Bronstein, 2001). Sensory system integration is crucial for standing posture and postural stability is necessary for performing functional activities and to avoid fall (Sibley, Straus, Inness, Salbach, \& Jaglal, 2013).

Vision is seen as an important factor in maintaining balance (Hunter \& Hoffman, 2001; Paulus, Straube, \&

\footnotetext{
* Address for correspondence: Milena Vagaja, Department of Natural Sciences in Kinanthropology, Faculty of Physical Culture, Palacký University Olomouc, třída Míru 117, 77111 Olomouc, Czech Republic. E-mail: milena.vagaja@gmail.com
}

Brandt, 1984; Redfern et al., 2001). It was reported that a well-functioning visual system complements the vestibular activities during standing posture (Rutkowska et al., 2015) and body sway can be reduced by almost $50 \%$ if the visual information is available (Turano, Dagnelie, \& Herdman, 1996). But different visual conditions such as following a cue or looking in one direction may increase (Kim, Moon, \& Cho, 2016) or decrease body sway (Schulmann, Godfrey, \& Fisher, 1987). The common approach in most of the recent studies exploring the visual control of posture is that a stable gaze (fixation), saccades or tracking (smoothpursuit) eye movements are used to understand the impact of different visual conditions on postural stability. For instance, the effects of fixations, saccades and/or smooth-pursuit eye movements on standing balance were analyzed in children during standard Romberg condition (Ajrezo, Wiener-Vacher, \& Bucci, 2013), and during various bipedal stance conditions in adults (Kim et al., 2016) and elderly (Aguiar et al., 2015). In addition, Rodrigues et al. (2013) conducted an experiment to analyze the influence of saccadic eye movements on postural control in young adults while standing upright. Participants stood in a wide stance (in alignment with the shoulders) and narrow stance (feet together) and were instructed to follow three 
conditions: fixation condition and saccadic conditions with low and high frequencies. The same experimental protocol with similar trial repetitions was used in older adults (Aguiar et al., 2015). While Kim et al. (2016) analyzed postural stability in three directions of smooth-pursuit conditions at three different speeds during stance on a flat surface, in Thomas, Bampouras, Donovan, and Dewhurst (2016) participants were balancing on a force platform under fixation, saccadic and smooth-pursuit visual conditions with a combination of fixed, absent and oscillating visual backgrounds. Also, postural control was measured on a force platform during fixation and horizontal saccades conditions in a study by Ajrezo et al. (2013). Legrand et al. (2013) manipulated with a two fixation conditions with and without distractors, prosaccades (reactive and voluntary saccades) and antisaccades while performing the Standard Romberg and Tandem-Romberg test.

Contradictory outcomes suggest that fixating a stable cue facilitates balance performance (Paulus et al., 1984; Schulmann et al., 1987), but also increases the excursion of the center of pressure (Ajrezo et al., 2013). It was also found that a postural sway is attenuated by a stationary fixation on a fixed background, in contrast to smooth pursuit eye movements and oscillating background that increases it (Thomas et al., 2016). On the contrary, saccadic eye movements were shown to reduce the magnitude of body sway (Aguiar et al., 2015; Legrand et al., 2013; Rodrigues et al., 2013, 2015) comparing to fixations tasks. However, controversial results in earlier researches might be due to various experimental protocols and variables that were used, such as different postural tasks (narrow or wide bipedal positions), different gaze tasks (directions, frequencies, amplitudes) or different age groups.

Stoffregen, Bardy, Bonnet, and Pagulayan (2006) mentioned two general predictions that could possibly explain underlying mechanisms used while performing a suprapostural (dual) task (such as visual task or reaction time task) during standing. So-called central competition, referring to competitive activities of central processing resources, may explain a decreased postural control while performing a suprapostural task, because both postural and suprapostural tasks use the same cognitive resources. Therefore, the combination of postural tasks with suprapostural activity may lead to either decreased postural performance, decreased suprapostural task, or both. On the other hand, they argue that postural control can be accommodated in order to support the realization of suprapostural activity. In this manner, with regards to the suprapostural visual task, it was suggested that body sway might be adjusted during a visual search to facilitate eye movements (Stoffregen et al., 2006; Stoffregen, Pagulayan,
Bardy, \& Hettinger, 2000). In other words, saccadic eye movements lead to decreased body sway to allow more accurate gaze shifts (Rodrigues et al., 2013, 2015; Stoffregen et al., 2006). This prediction is explained as the functional relationship between eye movements and body sway, as a very little change in a body sway may influence the accuracy of the eye movements (Stoffregen et al., 2006, 2000).

In addition to the preceding paragraph, we can expect that functional coupling between postural control and gaze behavior might be moderated by the demand of a task. Although, one can presume if a visual task is more challenging that a postural control might increase in order to support solving a visual task, we ask whether a more challenging visual task attenuates postural control during more demanding postural task. Moreover, in recent years previous studies were conducted on a stable/flat surface, during which, participants were standing upright. To this date, only Schulmann et al. (1987) conducted an experiment on a stability platform that was moving in the frontal plane. Therefore, the aim of the study was to examine the influence of saccadic eye movements on postural stability in healthy young adults while maintaining balance on an unstable platform.

\section{Methods}

\section{Participants}

A total number of thirty-two students (15 males, 17 females, age $22.8 \pm 2.7$ years, height $170.6 \pm 7.9 \mathrm{~cm}$, body mass $68.8 \pm 10.6 \mathrm{~kg}$ ) participated in this study. A-priori power analysis indicated that a sample size of 32 would be sufficient to detect a significant effect of the independent variables with repeated measurements within-subject design with a power of $.80, \alpha$ of .05 , assuming medium effect size of .25 and reliability of the measurement of at least .5. The computation was performed by G*Power 3.1.9.2 (Faul, Erdfelder, Lang, $\&$ Buchner, 2007). Subjects with medical conditions, such as visual impairment (including eye abnormalities), neurological impairment, musculoskeletal disorders, with complaints of dizziness or vertigo, or with similar comorbid conditions were not included in the measurement. Participation in the study was voluntary and without any incentives. Information about the purpose of the study was given to all participants before the experiment. The study was conducted after the approval by the Ethics Committee of the Faculty of Physical Culture, Palacký University Olomouc and participants signed written informed consent before testing. 


\section{Apparatus and procedures}

The main task was to maintain balance on the stabilometer (model 16030, Lafayette Instrument, Lafayette, IN, USA; sampling frequency $25 \mathrm{~Hz}$ ) positioned at a distance of $1.5 \mathrm{~m}$ from a screen onto which visual cues were projected (Hunter \& Hoffman, 2001). The platform tilts to the left and the right with a maximum deviation of $15^{\circ}$ on both sides and is used for maintaining balance, i.e., to be kept in a horizontal plane during the standing position. Participants wore a head-mounted eye tracker (SMI ETG 2W, SensoMotoric Instruments GmbH, Teltow, Germany; sampling frequency $30 \mathrm{~Hz}$ ), which is consisted of headgear (eye-glasses) and a battery pack that was put into a waist bag so that participants could move freely during the experiment. The eye movements were recorded binocularly with a gaze tracking accuracy of $0.5^{\circ}$ and a gaze tracking range of $80^{\circ}$ horizontal and $60^{\circ}$ vertical. Stabilometer and eye tracker were synchronized with a sound signalization to control the trials. The synchronization between stabilometer laptop and eye tracker SMI-ETG laptop was done with the parallel port interfacing through the peripheral component interconnect express card and line print terminal connector. Gaze was recorded through the iView ETG eye tracker software (Version 3.5; SensoMotoric Instruments $\mathrm{GmbH}$, Teltow, Germany). A transistor-transistor logic signals from the stabilometer software were received in the iView ETG eye tracker software at the beginning and the end of each trial. Using the "event $\log$ " in the iView software, each trigger line was controlled during each trial.

\section{Visual stimuli}

Visual cues were projected on the screen from the video projector Epson EB-96W (Seiko Epson Corporation, Suwa, Japan) installed above the stabilometer on the ceiling. During maintaining balance, participants were asked to follow five visual conditions: a) fixation condition, b) horizontal slow saccadic eye movements at the frequency of $0.5 \mathrm{~Hz}, \mathrm{c}$ ) horizontal fast saccadic eye movements at the frequency of $1.1 \mathrm{~Hz}, \mathrm{~d}$ ) vertical slow saccadic eye movements at the frequency of 0.5 $\mathrm{Hz}$, and e) vertical fast saccadic eye movements at the frequency of $1.1 \mathrm{~Hz}$ (Aguiar et al., 2015; Rodrigues et al., 2015). The stimulus was a black dot, in a diameter of $2 \mathrm{~cm}$ presented on the white screen in front of participants at the level of their eyes, which was adopted by the height of participants. In the fixation condition, the black dot appeared in the center of the screen. During the entire trial, the dot was on the screen and participants were asked to fixate it. During horizontal slow and horizontal fast saccadic conditions, participants were asked to follow the dots that were appearing and disappearing in the horizontal direction during the entire trial at the frequencies $0.5 \mathrm{~Hz}$ and $1.1 \mathrm{~Hz}$, respectively. Saccadic distance between the left and right dots was $29 \mathrm{~cm}$, i.e., $14.5 \mathrm{~cm}$ from the center on the left and the right side. The same procedure was followed for the vertical direction. In addition, both horizontal and vertical conditions were visually designed within the visual angle of $11^{\circ}$ (Aguiar et al., 2015; Rodrigues et al., 2015) in order to minimize head movement. Still, there were no restrictions to participants about their head movement during trials (Aguiar et al., 2015).

The experiment was conducted in the laboratory. The entire experiment consisted of 36 trials that were divided into 6 blocks. Each trial lasted $30 \mathrm{~s}$. The rest interval between each trial was $30 \mathrm{~s}$ and between each block $1 \mathrm{~min}$. Before the beginning of the experiment, each participant was required to wear sports shoes and to perform one practice trial in a duration of $15 \mathrm{~s}$ to become familiar with the task. After a practice trial, three-point calibration was done while the participant was instructed to fixate gaze at the central, horizontal and vertical dots/points presented in front on the screen. Calibration was done at the beginning of each block and after the third trial in each block. The system was re-calibrated if the points of regard deviation was more than $0.5^{\circ}$ of visual angle from the calibration points. The order of visual tasks was randomized e.g., the first block started with two central conditions, followed by two vertical conditions (slow then fast) and horizontal conditions (slow then fast), etc. In order to have an even number of repetitions in each block, fixation condition was repeated. The approximate time for each block was 6-8 min including approximate calibration time of 1-3 min. The measurement started when the participant stabilized a horizontal position.

\section{Data processing}

Data analysis

For the stabilometer data analysis, the procedure described by Shea and Wulf (1999) was followed to compute the root mean square error (RMSE). RMSE describes the deviation of the contact surface from the horizontal position identified as $0^{\circ}$. The higher the RMSE the less stable posture during the task indicating that RMSE is a measure of postural sway in mediallateral direction if used for assessment of postural stability during standing on stabilometer.

\section{Statistical analysis}

The mean of all trials in each condition was subjected to further analysis. The Kolmogorov-Smirnov test was used for the analysis of distribution confirming normal distribution for all variables. Repeated measures analysis of variance (ANOVA) was performed for the effect of conditions. Furthermore, the effect of speed 
of saccades and direction of saccades was also studied. For the investigation of effect of the speed of saccades, results obtained from trials in horizontal and vertical fast conditions and horizontal and vertical slow conditions were averaged to obtain three visual conditions fixation, fast saccades, slow saccades, which were again subjected to repeated measures. Similarly, for investigation of direction of saccades, results obtained during fast and slow horizontal and fast and slow vertical conditions were averaged to obtain another three visual conditions - fixation, vertical saccades, horizontal saccades - and subjected to repeated measures ANOVA. Analyses were performed at significance level $p<.05$ with effect size indicated by partial $\eta^{2}\left(\eta_{\mathrm{p}}^{2}\right)$ in software Statistica (Version 12; StatSoft, Tulsa, OK, USA).

\section{Results}

\section{Manipulation check}

An eye-tracking system was used for visual manipulation checks. The iView ETG eye tracker online system confirmed that participants were following the given instructions during all trials.

\section{Performance outcome}

When investigating effect of all visual conditions, no significant effect was found $\left(p=.340, \eta_{\mathrm{p}}^{2}=.036\right)$. For the effect of speed of saccadic eye movement, again no significant effect was found $\left(p=.251, \eta_{\mathrm{p}}^{2}=.044\right)$ with similar results obtained for the effect of directions of saccades $\left(p=.287, \eta_{\mathrm{p}}^{2}=.039\right)$. Descriptive results for all conditions can be found in Table 1 .

Table 1

Descriptive results for all visual conditions, different speeds and directions of saccades (stated as mean $\pm S D$ )

\begin{tabular}{lc}
\hline Visual stimuli & RMSE (deg) \\
\hline Fixation & $3.37 \pm 1.11$ \\
Horizontal slow saccades & $3.35 \pm 1.21$ \\
Horizontal fast saccades & $3.43 \pm 1.19$ \\
Vertical slow saccades & $3.43 \pm 1.19$ \\
Vertical fast saccades & $3.48 \pm 1.25$ \\
Fast saccades & $3.46 \pm 1.20$ \\
Slow saccades & $3.39 \pm 1.18$ \\
Horizontal saccades & $3.39 \pm 1.18$ \\
Vertical saccades & $3.46 \pm 1.20$ \\
\hline
\end{tabular}

Note. $\quad$ RMSE $=$ root mean square error.

\section{Discussion}

Contradictory results in earlier studies focusing on visual control of posture show the same eye movements' conditions have a different impact on postural stability. The present study assessed the influence of five different visual conditions (fixation, horizontal slow, horizontal fast, vertical slow and vertical fast eye movements) on standing posture. The results show that postural stability is not influenced by visual stimuli in healthy young adults while maintaining balance on an unstable platform.

One hypothesis related to an assumption that a body sway will decrease under a stable gaze (fixation) condition comes from the concept of saccadic suppression (Oblak, Gregoric, \& Gyergyek, 1985) suggesting that, during saccadic eye movements the information pick up from the optic array might be suppressed, which would be similar to a closed eyes condition, that is shown to increase imbalance (Stoffregen et al., 2006). Another explanation for a decreased body sway under fixation condition can be found in extraocular balance control, as it is shown that extraocular signals improve balance, because during postural sway eye movements used to maintain fixation provide information about body position relative to a target (Guerraz \& Bronstein, 2008; Thomas et al., 2016). Still, our results show no effect of the fixation task on postural sway relative to saccadic conditions. A potential reason may lay in a postural control strategy, as young adults might use sensory feedback information from the platform together with extraocular information from a visual target.

Contrary to previous findings showing body sway was attenuated during saccades (Aguiar et al., 2015; Legrand et al., 2013; Rodrigues et al., 2013, 2015), we found no differences in a postural sway during different saccadic conditions. It was argued, that a higher frequency of saccades plays an important role in a body sway decrease (Aguiar et al., 2015; Rodrigues et al., 2013, 2015; Thomas et al., 2016). Following the hypothesis that a postural control might be modulated in order to facilitate a gaze changes (Stoffregen, Bardy, Bonnet, \& Pagulayan, 2006), authors explain that during high frequencies saccades a feed-forward motor strategy is used (Aguiar et al., 2015; Thomas et al., 2016) that increases task complexity and, therefore, may explain a body sway decrease. But present findings show no impact of saccadic visual tasks at lower $(0.5 \mathrm{~Hz})$ nor higher $(1.1 \mathrm{~Hz})$ frequencies on participants' performance. That, on the other hand, may be a consequence of feed-forward motor control in which saccadic eye movements could be anticipated (Guerraz \& Bronstein, 2008; Thomas et al., 2016), therefore, may lower the effect of a task complexity. In addition, 
Rodrigues et al. (2013) discussed the frequency of saccadic eye movements as a factor that could differently affect a posture depending on a stance difficulty. Still, we may assume that in the present study, the postural task (maintaining balance on an unstable platform) was not challenging to healthy young participants.

\section{Conclusions}

In summary, this study provides evidence that postural control during upright standing is not influenced by secondary visual tasks in healthy young adults. The results are not in line with previous findings showing that saccadic eye movements help reducing body sway. Also, our results show no impact of different saccadic speeds nor different directions on a postural sway in young adults. Despite the fact that the experiment was designed to exclude head motion by establishing the exact visual angle, and therefore to possibly exclude vestibular effect, participants might have adopted a strategy that used more vestibular than visual information for maintaining balance. This could be interpreted as compensation that helps young adults maintain postural stability during standing on an unstable platform.

\section{Acknowledgments}

This study was supported by the Internal Grant Agency of Palacký University Olomouc (no. IGA_FTK_2018_013).

\section{Conflict of interest}

There were no conflicts of interest.

\section{References}

Aguiar, S. A., Polastri, P. F., Godoi, D., Moraes, R., Barela, J. A., \& Rodrigues, S. T. (2015). Effects of saccadic eye movements on postural control in older adults. Psychology \& Neuroscience, 8, 19-27.

Ajrezo, L., Wiener-Vacher, S., \& Bucci, M. P. (2013). Saccades improve postural control: A developmental study in normal children. PLOS One, 8, e81066.

Faul, F., Erdfelder, E., Lang, A. G., \& Buchner, A. (2007). $\mathrm{G}$ *Power 3: A flexible statistical power analysis program for the social, behavioral, and biomedical sciences. Behavior Research Methods, 39, 175-191.

Guerraz, M., \& Bronstein, A. M. (2008). Ocular versus extraocular control of posture and equilibrium. Clinical Neurophysiology, 38, 391-398.
Hammami, R., Behm, D. G., Chtara, M., Othman, A. B., \& Chaouachi, A. (2014). Comparison of static balance and the role of vision in elite athletes. Journal of Human Kinetics, 41, 33-41.

Han, J., Anson, J., Waddington, G., Adams, R., \& Liu, Y. (2015). The role of ankle proprioception for balance control in relation to sports performance and injury. BioMed Research International, 2015, 842804.

Hunter, M. C., \& Hoffman, M. A. (2001). Postural control: Visual and cognitive manipulations. Gait and Posture, 13, 41-48.

Kim, S., Moon, B., \& Cho, H. (2016). Smooth-pursuit eye movements without head movement disrupt the static body balance. Journal of Physical Therapy Science, 28, 1335-1338.

Latash, M. L. (2012). Fundamentals of motor control. Amsterdam, Netherlands: Elsevier.

Legrand, A., Mazars, K. D., Lazzareschi, J., Lemoine, C., Olivier, I., Barra, J., \& Bucci, M. P. (2013). Differing effects of prosaccades and antisaccades on postural stability. Experimental Brain Research, 227, 397-405.

Oblak, B., Gregoric, M., \& Gyergyek, L. (1985). Effects of voluntary eye saccades on body sway. In M. Igarashi \& F. O. Black (Eds.), Vestibular and visual control on posture and locomotor equilibrium (pp. 122-126). New York, NY: Karger.

Paulus, W., Straube, A., \& Brandt, T. (1984). Visual stabilization of posture. Brain, 107, 1143-1163.

Redfern, M. S., \& Furman, J. M. (1994). Postural sway of patients with vestibular disorders during optic flow. Journal of Vestibular Research, 4, 221-230.

Redfern, M. S., Yardley, L., \& Bronstein, A. M. (2001). Visual influences on balance. Journal of Anxiety Disorders, 15, 81-94.

Rodrigues, S. T., Aguiar, S. A., Polastri, P. F., Godoi, D., Moraes, R., \& Barela, J. A. (2013). Effects of saccadic eye movements on postural control stabilization. Motriz: Revista de Educacao Fisica, 19, 614-619.

Rodrigues, S. T., Polastri, P. F., Carvalho, J. C., Barela, J. A., Moraes, R., \& Barbieri, F. A. (2015). Saccadic and smooth pursuit eye movements attenuate postural sway similarly. Neuroscience Letters, 584, 292-295.

Rutkowska, I., Bednarczuk, G., Molik, B., Morgulec-Adamowicz, N., Marszałek, J., Kazmierska-Kowalewska, K., \& Koc, K. (2015). Balance functional assessment in people with visual impairment. Journal of Human Kinetics, 48, 99-109.

Schmidt, R. A., \& Lee, T. D. (2011). Motor control and learning: $A$ behavioral emphasis (5th ed). Champaign, IL: Human Kinetics.

Schulmann, D. L., Godfrey, B., \& Fisher, A. G. (1987). Effect of eye movements on dynamic equilibrium. Physical Therapy, 67, 1054-1059.

Shea, C. H., \& Wulf, G. (1999). Enhancing motor learning through external-focus instructions and feedback. Human Movement Science, 18, 553-571.

Sibley, K. M., Straus, S. E., Inness, E. L., Salbach, N. M., \& Jaglal, S. B. (2013). Clinical balance assessment: Perceptions of commonly-used standardized measures and current practices among physiotherapists in Ontario, Canada. Implementation Science, 8, 33. 
Stoffregen, T. A., Bardy, B. G., Bonnet, C. T., \& Pagulayan, R. J. (2006). Postural stabilization of visually guided eye movements. Ecological Psychology, 18, 191-222.

Stoffregen, T. A., Pagulayan, R. J., Bardy, B. G., \& Hettinger, L. J. (2000). Modulating postural control to facilitate visual performance. Human Movement Science, 19, 203-220.
Thomas, N. M., Bampouras, T. M., Donovan, T., \& Dewhurst, S. (2016). Eye movements affect postural control in young and older females. Frontiers in Aging Neuroscience, 8, 216.

Turano, K. A., Dagnelie, G., \& Herdman, S. J. (1996). Visual stabilization of posture in persons with central visual field loss. Investigative Ophthalmology and Visual Science, 37, 1483-1491. 